\title{
Ischemia/Reperfusion Injury in the Aged Liver: The Importance of the Sinusoidal Endothelium in Developing Therapeutic Strategies for the Elderly
}

\author{
Diana Hide, $\mathrm{PhD},{ }^{1,2,0}$ Alessandra Warren, $\mathrm{PhD}^{3}$ Anabel Fernández-Iglesias, $\mathrm{PhD},{ }^{1,2}$ \\ Raquel Maeso-Díaz, MSc, ${ }^{1}$ Carmen Peralta, PhD, ${ }^{2,4}$ David G. Le Couteur, PhD, ${ }^{3, \infty}$ \\ Jaime Bosch, MD, PhD, ${ }^{1,2,5}$ Victoria C. Cogger, $\mathrm{PhD}^{3}$ and Jordi Gracia-Sancho, $\mathrm{PhD}^{1,2,5, *, 0}$
}

'Liver Vascular Biology Research Group, Barcelona Hepatic Hemodynamic Laboratory, IDIBAPS Biomedical Research Institute, University of Barcelona Medical School, Barcelona, Spain. ${ }^{2}$ Biomedical Research Network Center in Hepatic and Digestive Diseases (CIBEREHD), Madrid, Spain. ${ }^{3}$ Center for Education and Research on Ageing \& ANZAC Research Institute, University of Sydney and Concord Hospital, Australia. ${ }^{4}$ Protective Strategies Against Hepatic Ischemia-Reperfusion Group, IDIBAPS, Barcelona, Spain. ${ }^{5} \mathrm{Hepatology}$, Department of Biomedical Research, Inselspital, Bern University, Switzerland.

*Address correspondence to: Jordi Gracia-Sancho, PhD, Liver Vascular Biology Research Group, Institut d'Investigacions Biomèdiques August Pi i Sunyer (IDIBAPS), Rosselló 149, 4th Floor, 08036 Barcelona, Spain. E-mail: jordi.gracia@idibaps.org

Received: September 20, 2018; Editorial Decision Date: January 4, 2019

Decision Editor: Rozalyn Anderson, PhD

\begin{abstract}
The liver endothelium plays a key role in the progression and resolution of liver diseases in young and adult individuals. However, its role in older people remains unknown. We have herein evaluated the importance of the sinusoidal endothelium in the pathophysiology of acute liver injury, and investigated the applicability of simvastatin, in aged animals. Eighteen-months-old male Wistar rats underwent 60 minutes of partial warm ischemia followed by 2 hours of reperfusion (WIR). A group of aged rats received simvastatin for 3 days before WIR. Endothelial phenotype, parenchymal injury, oxidative and nitrosative stress, and fenestrae dynamics were analyzed. The effects of WIR and simvastatin were investigated in primary LSEC from aged animals. The results of this study demonstrated that WIR significantly damages the liver endothelium and its effects are markedly worse in old animals. WIR-aged livers exhibited reduced vasodilation and sinusoidal capillarization, associated with liver damage and cellular stress. Simvastatin prevented the detrimental effects of WIR in aged livers. In conclusion, the liver sinusoidal endothelium of old animals is highly vulnerable to acute insult, thus targeted protection is especially relevant in preventing liver damage. Simvastatin represents a useful therapeutic strategy in aging.
\end{abstract}

Keywords: Liver sinusoidal endothelial cells, LSEC, Hepatic hemodynamic, Intrahepatic vascular resistance, Statins

Ischemia/reperfusion injury is caused by an initial interruption of blood supply, followed by the restoration of perfusion. The impact of the cessation and reestablishment of blood supply can result in a cascade of pathological impacts, such as oxidative stress, release of prothrombotic cytokines and apoptosis (1). Clinically, warm ischemia-reperfusion (WIR) injury is almost unavoidable in liver resection surgery, transplantation, and in blood transfusion after hemorrhagic shock, and may contribute to delayed graft function and liver failure. In addition to the well-defined parenchymal injury $(2,3)$, we recently demonstrated that ischemia reperfusion causes an acute deterioration of the hepatic microvascular function both in cold and warm ischemia conditions (4-6).

In accordance with the current population pyramids, most of the clinical situations involving hepatic WIR occur in people aged more than 60 and, although we have not yet fully elucidated the mechanisms it has been previously shown that older livers are more susceptible to pathological stress responses, have decreased regenerative capacity, and this results in increased morbidity and mortality (712). A key factor in our limited understanding of the mechanisms involved in the age-related loss of resilience to ischemia-reperfusion 
injury may be that the majority of the present understanding derives from experiments conducted in young animals (aging 6-16 weeks) with only very few studies evaluating the effects of acute liver injury in older livers $(13,14)$. The initial point of impact of ischemia-reperfusion injury in the liver is the liver sinusoid, a highly differentiated and specialized vascular bed. Lining these vessels are the liver sinusoidal endothelial cells (LSEC), which have major physiological roles in homeostasis, immune function, and facilitating bi-directional transfer of substances between blood and the space of Disse via fenestrations (15-18). With aging, significant ultrastructural and functional changes in LSEC are produced conducing to a phenomenon termed pseudo-capillarization $(19,20)$. Importantly, a recently published study from our group deeply characterized the hepatic sinusoid in aging demonstrating that healthy aged livers exhibit slight but significant de-regulation in hepatic and microcirculatory functions, which may result in a higher vulnerability in the face of acute injury (21).

Statins, HMG-CoA inhibitors designed to lower cholesterol levels, have many pleiotropic effects independent of lipid lowering (22). Administration of simvastatin, one of the most widely evaluated statins, has demonstrated its effectiveness protecting the liver sinusoidal endothelium in experimental models of chronic and acute liver injury in young animals (4,6,23-25). However, it's utility in acute liver injury in older is completely unknown.

This study was designed to better understand the effects of acute liver injury on aged rats, in particular focusing on sinusoidal endothelial phenotype and liver ultrastructure; and to further investigate the applicability of statins preventing liver injury in aging.

\section{Methods}

\section{Animals and Treatment}

Male Wistar rats aging 18 months (Janvier Laboratories, Le GenestSaint-Isle, France) were treated with simvastatin $(25 \mathrm{mg} / \mathrm{kg}$ body weight) or its vehicle by gavage once a day for 3 days. Dosing of statin was based on previous literature from our team and others $(23,25,26)$. Animals were kept in environmentally controlled animal facilities at the Institut d'Investigacions Biomèdiques August Pi i Sunyer (IDIBAPS). All experiments were approved by the Laboratory Animal Care and Use Committee of the University of Barcelona and were conducted in accordance with European Community guidelines for the protection of animals used for experimental or other scientific purposes (EEC Directive 86/609).

\section{Liver Vascular Studies}

Under anesthesia with intraperitoneal ketamine $(100 \mathrm{mg} / \mathrm{kg}$, Merial Laboratories, Barcelona, Spain) and midazolam (5 mg/kg, Normon, Tres Cantos, Madrid, Spain) partial warm ischemia affecting $70 \%$ of liver volume was induced by clamping the portal triad irrigating the medial and left lateral lobes with an atraumatic clamp for 1 hour, which was followed by 2 hours of reperfusion (27). Sham operated animals were included. After the WIR period, liver microvascular response was assessed in the isolated, in situ liver perfusion system, as described (28). Livers were perfused at a constant portal flow of $35 \mathrm{~mL} / \mathrm{min}$ and after 20 minutes of stabilization, liver endothelial function was evaluated analyzing endothelium-dependent vasorelaxation to incremental doses of acetylcholine (Ach; $10^{-7}$ to $10^{-5} \mathrm{M}$ ) after pre-constriction with methoxamine $\left(10^{-4} \mathrm{M}\right)$.

At the end of the study, liver samples from lobules that suffered WIR were stored for molecular analysis as described below.

\section{Electron Microscopy}

After the experimental procedure, 5-7 livers per group were perfused through portal vein with a fixation solution containing $2.5 \%$ glutaraldehyde and $2 \%$ paraformaldehyde in $0.1 \mathrm{M}$ cacodylate buffer $0.1 \%$ sucrose and fixed overnight at $4^{\circ} \mathrm{C}$. Samples were washed three times with $0.1 \mathrm{M}$ sodium cacodylate buffer. Liver sections were post-fixed with $1 \%$ osmium in cacodylate buffer and dehydrated in an ethanol or acetone gradient to $100 \%$.

For scanning electron microscopy 6-8 liver blocks per sample were mounted on stubs, sputter coated with platinum and examined using a Jeol 6380 scanning electron microscope (JEOL Ltd, Tokyo, Japan). At least 10 images per animal were taken.

Measurements of fenestrae size, number, and density were carried out. Fenestrations were defined as open pores with diameters $<300 \mathrm{~nm}$. Diameter was defined as the major length of each fenestration or gap. Porosity was defined as the sum area of fenestrations/ total quantified area. Frequency was defined as number of fenestrae per $\mu \mathrm{m}^{2}$ (29).

For transmission electron microscopy, fixed liver tissue was embedded in Spurr resin, cut in $50 \mathrm{~nm}$ ultrathin sections, counterstained with uranyl acetate and lead citrate and examined using Philips CM10 transmission electron microscope. Ten micrographs per sample were taken to estimate \% of necrotic hepatocytes and $\%$ of sinusoids presenting each of the evaluated parameters (21).

\section{Histological Analysis}

Liver samples were fixed in $10 \%$ formalin, embedded in paraffin, sectioned, and slides were stained with hematoxylin and eosin (H\&E) to analyze the hepatic parenchyma (30). Hepatic histology was scored by two blinded independent researchers as previously described (31). Briefly, cytoplasmic vacuolation, nuclear pyknosis, cytoplasmic hypereosinophilia, loss of intercellular borders, necrosis and fat accumulation were scored as 0 -abstent, 1-focal, or 2general, and neutrophil infiltration as 0 -absent or 1 - present, making a maximum final score of 13 points.

\section{Immunohistochemical Stainings}

Liver samples were fixed in $10 \%$ formalin, embedded in paraffin and sectioned. After antigen retrieval procedure and endogenous peroxidase activity inhibition, sections were incubated with antivon Willebrand Factor (1:400; Dako, Glostrup, Denmark), anti-aSMA (1:1,000; M0851, Dako), anti-CD68 (1:100; BioRad, Paris, France) or anti-CD163 (1:100; BioRad) 1 hour at room temperature. HRP-Rabbit/Mouse (Dako) secondary antibody was added. Color development was induced by incubation with a DAB kit (Dako) and the sections were counterstained with hematoxylin. Sections were dehydrated and mounted. The specific staining was visualized and images were acquired using a microscope equipped with a digital camera and the assistance of Axiovision software. vWF relative volume was determined by point-counting morphometry on immunoperoxidase-stained sections, using a point grid to obtain the number of intercepts over vWF positive cells over the tissue. Six fields per liver were counted and the relative volume was calculated by dividing the number of points positive in sinusoidal areas by the total number of points over liver tissue (6). For CD68 and CD163, 10 images per sample were obtained and positive cells per field were counted. All analyses were performed blindly by two independent researchers. 


\section{Nitric Oxide Determination}

Levels of cGMP, surrogate marker of NO bioavailability, were analyzed in liver homogenates using an enzyme immunoassay following manufacturer instructions (Cayman Chemical Co., Ann Arbor, MI) (4).

\section{Nitrotyrosine Fluorohistochemistry}

Quantitative tyrosine nitration detection was performed as previously described (5). Briefly, slides were deparaffinised, hydrated, incubated with aqueous sodium dithionite solution $(10 \mathrm{mM})$ for $10 \mathrm{~min}$, washed with distilled water and then incubated overnight at $4^{\circ} \mathrm{C}$ with an equimolar solution of $\mathrm{AlCl}_{3}$ and salicylaldehyde $(200 \mathrm{mM})$. Afterwards, the aqueous solution was removed and sections were mounted in Fluoromount G medium (Southern Biotech, Birmingham, AL). Negative controls were included. Fluorescence images were obtained with a fluorescence microscope and quantitative analysis of at least six images per sample was performed with Image J $1.44 \mathrm{~m}$ software (National Institutes of Health, Bethesda, MD).

\section{Real-Time Quantitative PCR}

RNA from liver tissue was extracted using Trizol (Life Technologies), quantified using Nanodrop software (ND1000, Marshall Scientific, Hampton, $\mathrm{NH}$ ) and reverse transcribed to cDNA using Quantitect Reverse Transcription Kit (205311, Qiagen) previous elimination of genomic DNA of the sample. cDNA templates were amplified by SYBR green using the following primers (a-SMA: F，CTCATGCCATCATGCGTCTG; R, CACGCTCAG CAGTAGTCACG; Col1a1: F， GTACATCAGCCCAAACCCCA; R, TCGCTTCCATACTCGAACTGG; Col1a2: F, TCGCTTCCAT ACTCGAACTGG; R， GATGGCCTTTCTCACCAGGTT; pdgfrb: F, GTCAATGTCCCTGTCCGTGT; R, GTGTGGGTGACAG TTTTCGC; desmin: F, CAACTTCCGAGAAACCAGCC; R, CGTGTCTGGCTTACAGCACT; tnfa: F， ATGGGCTCCCT CTCATCAGT; R, GCTTGGTGGTTTGCTACGAC; il6: F, TCCGGAGAGGAGACTTCACA; R, GCCATTGCACAACT CTTTTCT; gapdh: F， GGCATCGTGGAAGGGCTCAT; R, AGGGATGATGTTCTGGGCTGC). $C_{t}$ values were normalized to those of GAPDH and expressed as relative changes versus the control $\left(\Delta \Delta \mathrm{C}_{\mathrm{t}}\right.$ method). All PCR reactions were performed in duplicate and using nuclease-free water as controls.

\section{Liver Sinusoidal Endothelial Cells Isolation and Treatments}

Rat liver sinusoidal endothelial cells (LSEC) were isolated by collagenase perfusion and Percoll density gradient as described (28) Highly pure and viable cells were used.

After 2 hours of isolation, LSEC were washed with phosphatebuffered saline (PBS) and treated with simvastatin $(1 \mu \mathrm{M})$ or its vehicle (DMSO $0.1 \%$ ) for 1 hour. After the treatment LSEC were incubated for 1 hour in an anoxic chamber (BD GasPak, BD Life Sciences), and afterwards removed from the chamber and immediately placed in the cell-culture incubator for reoxygenation during 2 hours.

Cells were processed for in situ analysis of intracellular levels of superoxide $\left(\mathrm{O}_{2}^{-}\right)$and nitric oxide (NO), or for SEM, as described below.

\section{LSEC Electron Microscopy}

Scanning electron microscopy on the cultured LSEC was performed as described (29). After the experimental procedure, LSEC were fixed in $2.5 \%$ glutaraldehyde in a $0.1 \mathrm{M}$ sodium cacodylate buffer. Cells on coverslips were osmicated $\left(1 \% \mathrm{OsO}_{4}\right.$ in $0.1 \mathrm{M}$ cacodylate buffer), dehydrated in an ethanol gradient to $100 \%$ and incubated for 2 minutes in hexamethyldisilazane. Coverslips were mounted on stubs, sputter coated with gold and examined using a Jeol 6380 scanning electron microscope (JEOL Ltd, Tokyo, Japan).

\section{LSEC Actin Filament Staining}

Actin filament structures were stained in primary LSEC with the Phalloidin Fluorescein Isothiocyanate Labelled (P582, SigmaAldrich) as described (32). Briefly, LSEC were fixed for 10 minutes in $4 \%$ paraformaldehyde in PBS, permeabilized for 10 minutes with $0.1 \%$ TRITON X-100 and stained with a $50 \mu \mathrm{g} / \mathrm{mL}$ fluorescent phalloidin conjugate solution for 30 minutes at $37^{\circ} \mathrm{C}$. LSEC were visualized using a Leica DM2500 Confocal Microscope (Leica Ltd, Wetzlar, Germany) and cellular cytoskeleton organization was evaluated qualitatively.

\section{DHE and DAF Staining}

In situ $\mathrm{O}_{2}{ }^{-}$and $\mathrm{NO}$ levels in LSEC were assessed with the fluorescent dyes dihydroethidium (DHE $10 \mu \mathrm{M}$; Molecular Probes, Inc., Eugene, OR) or 4-amino-5-methylamino-2',7'-difluorofluorescein diacetate (DAF-FM-DA $10 \mu \mathrm{M}$; Molecular Probes, Inc.) respectively as described $(33,34)$. Fluorescence images were obtained with a fluorescence microscope (Olympus BX51, Tokyo, Japan), and quantitative analysis of at least 20 images per condition was performed with Image J $1.44 \mathrm{~m}$ software.

\section{Western Blotting}

Liver samples were processed and western blot performed as described (28). Used primary antibodies: Nrf2 (Santa Cruz Biotech, Santa Cruz, CA), HO-1 (Enzo Life Sciences, Farmingdale, NY), phosphorylated eNOS at Ser1177 (Cell Signaling, Danvers, MA), total eNOS (BD Transduction Laboratories, Lexington, KY), VEGF (Abcam, Cambridge, UK), Flk1 (Santa Cruz Biotech, Santa Cruz, CA), phosphorylated myosin light chain at Ser19 (p-MLC, Cell Signaling) and phosphorylated moesin at Thr558 (Santa Cruz Biotech) all 1:1,000. Blots were revealed by chemiluminescence and protein expression was determined by densitometric analysis using the Science Lab 2001 Image Gauge (Fuji Photo Film, Düsseldorf, Germany). Blots were also assayed for GAPDH (1:5,000, SigmaAldrich) content as standardization of sample loading.

\section{Statistical Analysis}

Statistical analyses were performed with the IBM SPSS Statistics 20 for Windows statistical package. All results are expressed as mean \pm standard error of the mean. Comparisons between groups were performed with analysis of variance followed by LSD post hoc test, Student's $t$ test or nonparametrical tests when adequate. Differences were considered significant at $p<.05$.

\section{Results}

\section{Effects of WIR on Microcirculatory Phenotype and Function in Old Livers}

Liver warm ischemia followed by a 2-hour-reperfusion period promoted the development of microvascular dysfunction in old animals as evidenced by their reduced response to the endothelialdependent vasodilator acetylcholine (Figure 1A). Importantly, old 
livers undergoing acute injury exhibited significantly worse impairment in microvascular function compared with young animals (6). This microvascular dysfunction in older rats was further confirmed by a fourfold increase in the sinusoidal expression of $\mathrm{vWF}$, a well-accepted marker of LSEC capillarization (Figure 1B, top). Histological examination revealed that livers from older rats that had undergone WIR had significant hepatocellular damage including cytoplasmic vacuolization, neutrophil infiltration, and necrotic hepatocytes (Figure 1B, bottom). Biochemical analyses further demonstrated WIR-derived liver damage; while AST and ALT levels where 88 and $65 \mathrm{UI} / \mathrm{mL}$ in blood from sham animals these values raised to 2,420 and $4,669 \mathrm{UI} / \mathrm{mL}$, respectively, in the WIR group. Liver ultrastructure characterization using transmission electron microscopy confirmed that WIR caused loss of hepatic microvilli, enlargement of the Space of Disse, gap formation in the endothelium, peliosis (red blood cells in the space of Disse or parenchyma) and hepatocyte necrosis (Figure 1C).

Old rats that underwent pretreatment with simvastatin for 3 days exhibited attenuated WIR-associated microcirculatory derangements as indicated by improved sinusoidal function and vWF sinusoidal expression (Figure 1A and B). Furthermore, simvastatin treatment before WIR significantly reduced the hepatocellular damage observed by histological and ultrastructural examination (Figure 1B and C) but was not able to normalize liver enzymes (AST: 2,216 UI/mL, ALT: 5,926 UI/mL).

\section{De-regulation of Oxidative and Nitrosative Stress in Aged Livers Undergoing WIR}

WIR promoted a dramatic de-regulation in the intrahepatic oxidative and nitrosative stress. Indeed, acute liver injury led to an increment in intrahepatic NO, as suggested by the raise in the levels of its surrogate marker cGMP (Figure 2A), which was accompanied by higher nitrotyrosine formation, indicative of increased peroxynitrite production (Figure $2 \mathrm{~B}$ ), and reduced levels of the antioxidant transcription factor Nrf2 (Figure 2C). These changes were associated with a reduction in the expression of the active form of endothelial nitric oxide synthase (eNOS) (Figure 2D).

Simvastatin prevented the increments in $\mathrm{NO}$ and nitrotyrosines (Figure 2A and B), maintained the expression of $\mathrm{Nrf} 2$ and potentiated the antioxidant enzyme HO-1 (Figure 2C and E), while no changes in p-eNOS were observed (Figure 2D).

Nitric oxide and oxidative stress were also evaluated in LSEC isolated from vehicle- or simvastatin-treated aged rats undergoing in vitro anoxia and reoxygenation. We observed that $\mathrm{O}_{2}{ }^{-}$levels were reduced in the simvastatin-treated group (Figure $2 \mathrm{~F}$ ), thus confirming what was observed in liver tissue. Interestingly, LSEC from the simvastatin-treated group exhibited increased NO levels compared with vehicle cells (Figure $2 \mathrm{G}$ ), suggesting an improvement in intracellular endothelial NO availability.

\section{Characterization of Sinusoidal Cells Phenotype in Aged Livers Undergoing WIR}

Scanning electron microscopy (SEM) analysis of liver tissue and primary cultured LSEC revealed that acute liver injury causes a significant reduction in fenestrae frequency, without changes in diameter, which conduces to a reduced porosity (the \% of area covered by fenestrae) (Figure 3A and B). The beneficial effects of simvastatin on endothelial phenotype of 18-month-old livers following WIR was further supported by the observed maintenance of fenestrae number and endothelial porosity (Figure $3 \mathrm{~A}$ and $\mathrm{B}$ ).
Interestingly, maintenance of LSEC fenestrae in response to simvastatin was accompanied by reorganization of cellular cytoskeleton, as suggested by actin staining (Figure 3C). While vehicle-treated cells from WIR exposed livers exhibited an accumulation of abnormal cortical actin structures, which looked like small crescents or aggregates, LSEC treated with simvastatin maintained an organized cytoskeleton.

Due to the impact of the vascular endothelial growth factor (VEGF) on actin filament organization (35), this pathway was evaluated in liver tissue for the potential role in the fenestrae changes observed here. As seen in Figure 3D, hepatic VEGF protein expression was increased in simvastatin-treated aged animals, while its receptor Flk-1 was significantly reduced (Figure 3E). An additional mechanism for fenestrae regulation, namely contraction of actin cytoskeleton via rho-kinase (36), was also evaluated by p-MLC and $\mathrm{p}$-moesin protein determination in liver tissue, but there were no differences between both groups undergoing WIR (data not shown).

Analysis of hepatic stellate cells (HSC) and hepatic macrophages (HMФ) phenotype was performed by evaluating the protein and mRNA expression of specific markers in liver tissue. The results did not evidence any effect of simvastatin treatment on HSC phenotype (no changes in a-SMA, collagen 1a1, collagen 1a2, pdgfrb and desmin; data not shown). Contrarily, when analyzing the changes suffered by HМФ, we observed a change in morphology from spindle to oval shaped and a trend to increased CD163 positive cells in vehicle+WIR compared with sham animals (Supplementary Figure 1A). Simvastatin preserved the spindle-like morphology in some of the individuals and prevented the increase in CD163 positive cells. At mRNA level, TNF $\alpha$ expression was reduced in simvastatintreated animals without changes in IL-6 (Supplementary Figure 1B).

\section{Discussion}

Chronological age represents one of the most significant risk factors for the development of many diseases, such as liver disease, cardiovascular disease, and diabetes. It is now widely understood that intervention in the pathways involved in aging may have significant impact on the prevention of these age related conditions.

A recent study from our group described that healthy aging is associated with mild hepatic microcirculatory dysfunction, endothelial de-differentiation and a moderate activation of hepatic stellate cells (21). This study indicated that even if those changes are asymptomatic, the livers of older individuals may have less protective mechanisms against liver injury compared with young individuals. To further explore this hypothesis, we performed the present study evaluating the effects of an acute liver injury, as can be warm ischemia/reperfusion, in aged animals and proposed a vasoprotective strategy for this specific subpopulation.

The first observation derived from our study is that even if there is not a significant difference in liver endothelial function comparing sham-operated young and old animals, the effects of WIR on liver microcirculatory function is markedly worse in the aged ones. Indeed, our previous results studying hepatic WIR in young animals evidenced the development of acute circulatory dysfunction after 1 hour of warm ischemia followed by 2 hours of reperfusion (6). However, we herein show that applying the same experimental procedure to 18 -month-old rats causes much more severe microcirculatory dysfunction determined by the reduced response to the vasodilator acetylcholine, which is accompanied by increased sinusoidal vWF expression. In addition, histological and ultrastructural 
A

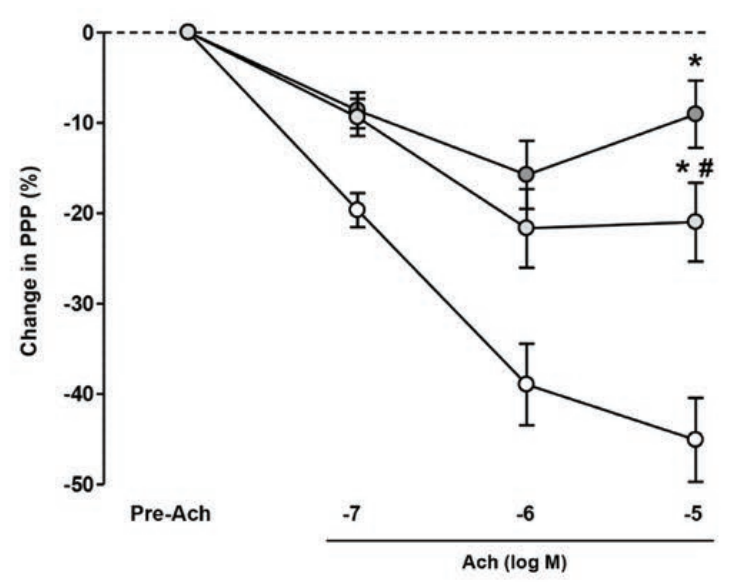

B

Sham
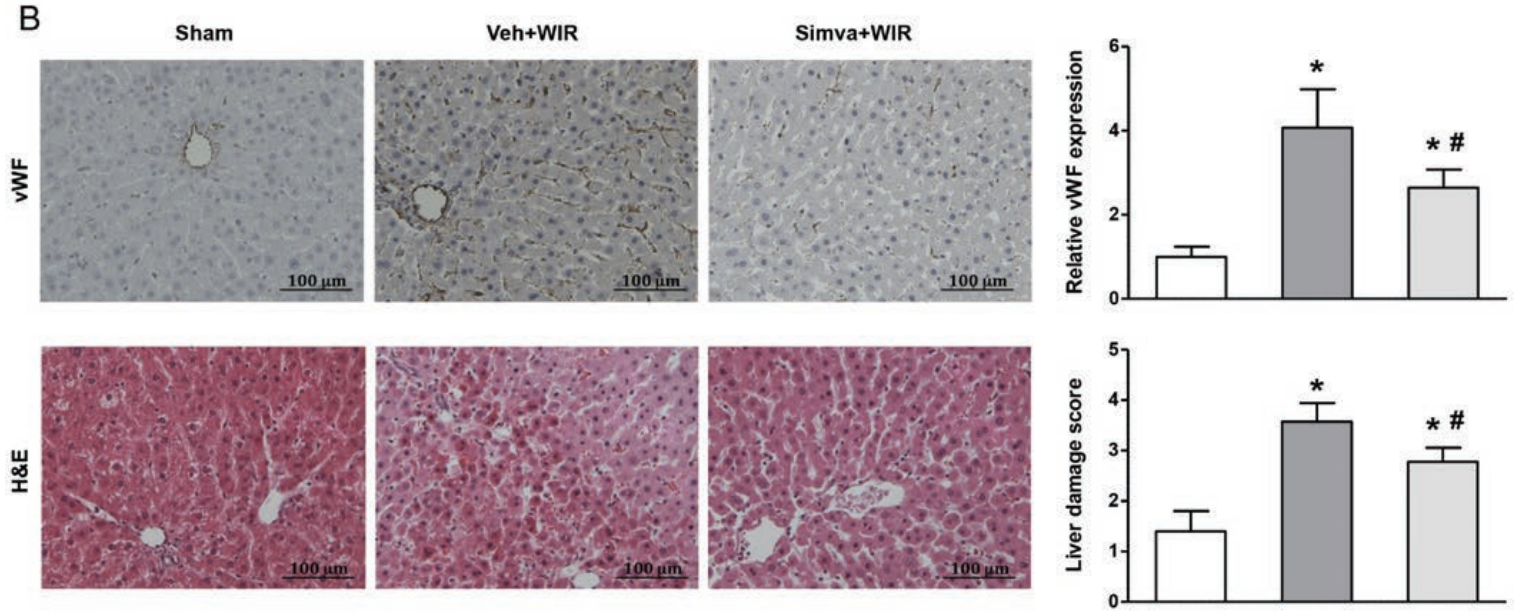

C

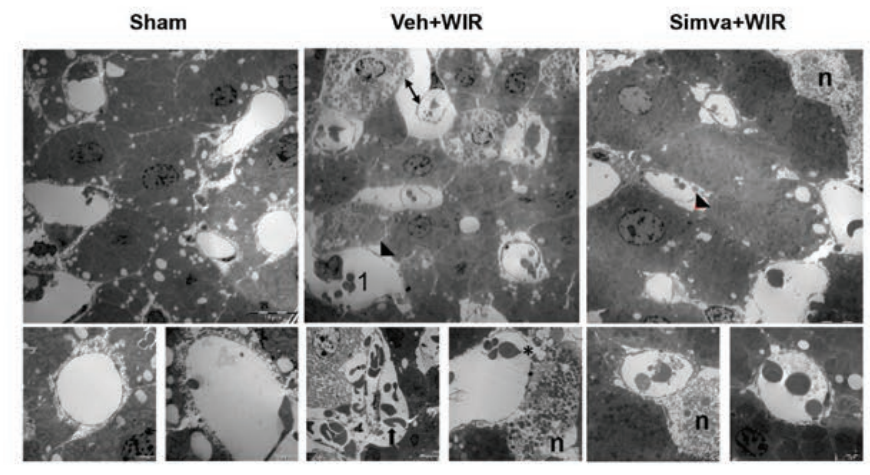

\begin{tabular}{cccccc}
\hline & Lack microvilli (\%) & $\begin{array}{c}\text { Enlarged Space } \\
\text { of Disse (\%) }\end{array}$ & Peliosis (\%) & $\begin{array}{c}\text { Gaps } \\
\text { endothelium } \\
(\%)\end{array}$ & $\begin{array}{c}\text { Necrotic } \\
\text { hepatocytes } \\
(\%)\end{array}$ \\
\hline Sham & $2.6 \pm 2.1$ & $0.8 \pm 0.8$ & $0.4 \pm 0.4$ & $5.6 \pm 1.7$ & $1.6 \pm 1.2$ \\
Veh+WIR & $84 \pm 4^{*}$ & $50 \pm 6^{*}$ & $43 \pm 5^{*}$ & $29 \pm 4^{*}$ & $26 \pm 4^{*}$ \\
Simva+WIR & $59 \pm 6$ & $41 \pm 6^{\#}$ & $32 \pm 5$ & $19 \pm 4^{\#}$ & $17 \pm 4$ \\
\hline
\end{tabular}

Figure 1. Warm ischemia and reperfusion markedly deteriorates liver microcirculation and hepatic viability in aged animals. (A) Evaluation of liver microcirculatory function as response to incremental doses of acetylcholine in aged animals treated with simvastatin, or vehicle, undergoing 1 hour of $70 \%$ hepatic warm ischemia followed by 2 hours of reperfusion, or sham-operation. (B) Sinusoidal vWF protein expression (top), and H\&E staining and semi-quantitative analysis of liver damage (bottom) in aged livers described in $\mathbf{A}$. Representative images $\times 20$. (C) Top: transmission electron micrographs of aged rats described in $\mathbf{A}$ (original magnification $\times 1,700$ and $\times 6,000$; arrowhead: lack of microvilli, double-headed arrow: enlarged space of Disse, asterisk: gaps in endothelium, arrow: peliosis, n: necrotic hepatocytes). Bottom table: \% of total sinusoids presenting each of the evaluated parameters. Values represent mean \pm standard deviation; $n=8$ animals per group $(\mathbf{A}$ and $\mathbf{B}), n=3$ animals per group $(\mathbf{C}) ;{ }^{*} p<.05$ vs sham, ${ }^{\#} p<.05$ vs vehicle; PPP $=$ portal perfusion pressure. 
A

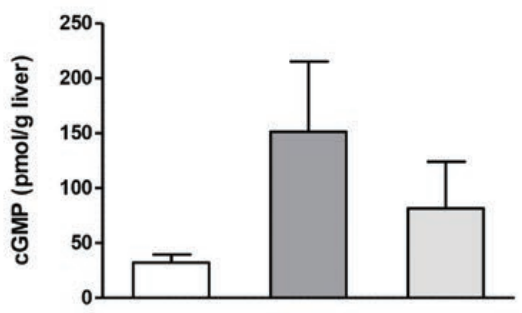

C
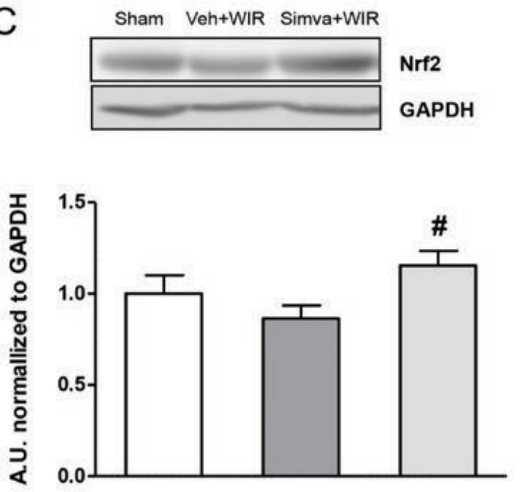

F
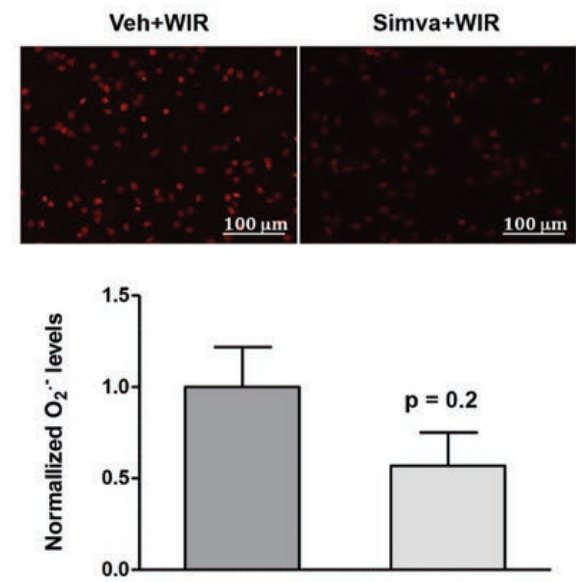

B

D
Sham

Veh+WIR

Simva+WIR
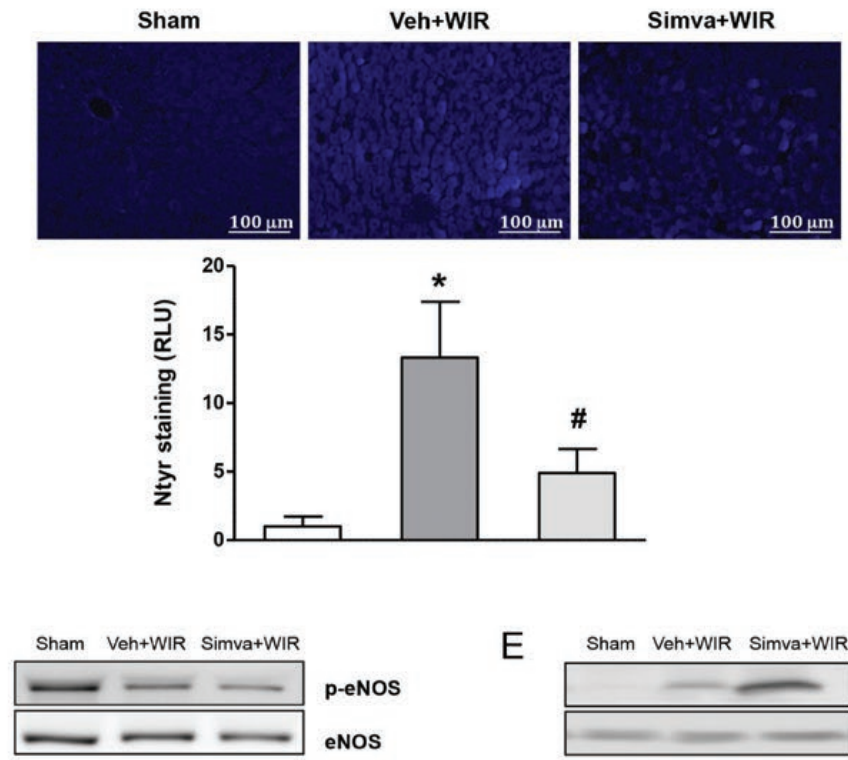

E
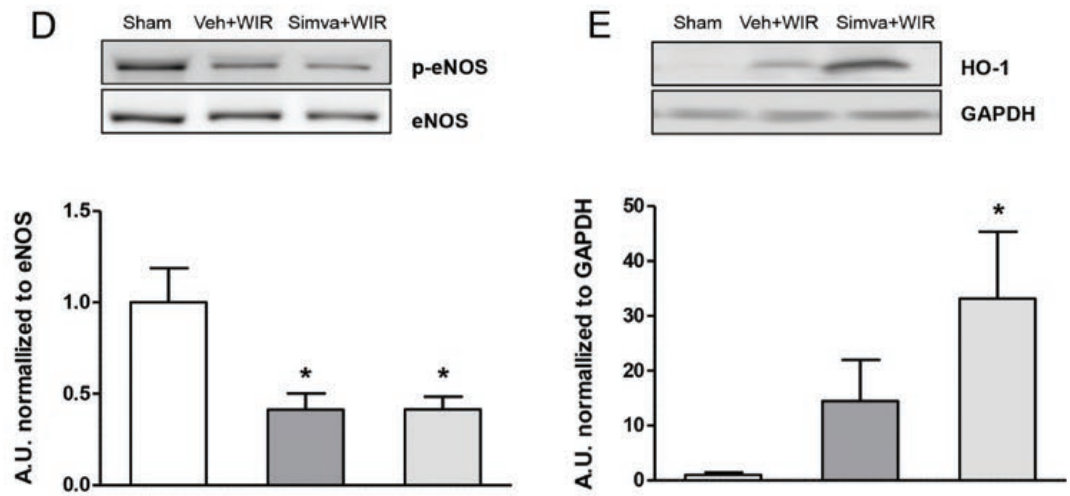

G
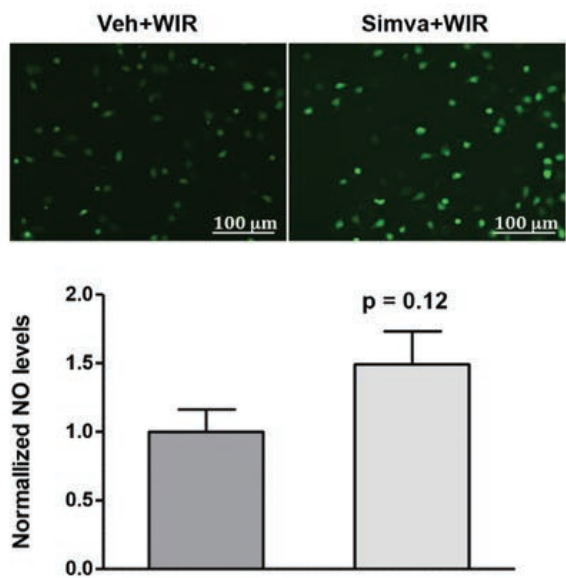

Figure 2. Warm ischemia and reperfusion de-regulates the oxidative stress and nitric oxide pathways in aged livers. (A) cGMP levels in liver tissue from aged rats pretreated with simvastatin, or vehicle, undergoing 1 hour of partial ischemia followed by 2 hours of reperfusion, or sham operation. (B) Nitrotyrosinated proteins fluorohistochemistry from livers described in A. (C) Representative images of Nrf2 and its corresponding densitometric analysis normalized to GAPDH from livers described in A. Results are referred to sham group. (D) Representative western blots of phosphorylated eNOS (p-eNOS) and total eNOS and corresponding quantification. (E) Representative western blots of heme oxygenase-1 (HO-1) and corresponding quantification. (F) Endothelial oxidative stress assessed as superoxide radical levels $\left(\mathrm{O}_{2}{ }^{-}\right.$, $)$determined in primary LSEC pretreated with simvastatin or vehicle and cultured under anoxia/reoxygenation conditions. Fluorescent intensity was divided by total number of cultured cells. (G) Endothelial nitric oxide (NO) levels from cells described in F. Fluorescent intensity was divided by total number of cultured cells. Representative images $\times 20 ; n=8$ animals per group (A-E), $n=3$ animals per group (F-G); ${ }^{*} p<.05$ vs sham, ${ }^{*} p<.05$ vs vehicle).

analysis revealed that WIR caused significant liver damage, both in liver sinusoids and parenchymal cells.

Statins exert cholesterol-independent hepatoprotective beneficial effects in preclinical and clinical scenarios of liver diseases, such as cirrhosis and bacterial infection (23,25,37-39). Nevertheless, very little is known about the effects of statins in older animal models. In fact, a single experimental study evaluated the effects of simvastatin in livers from healthy aged rats (40), showing positive impacts 
A
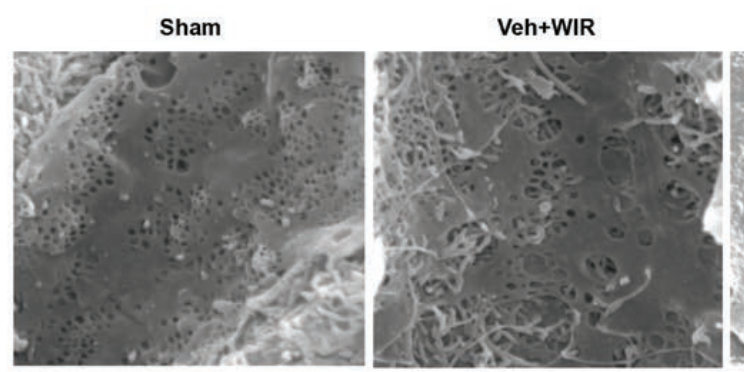

Simva+WIR
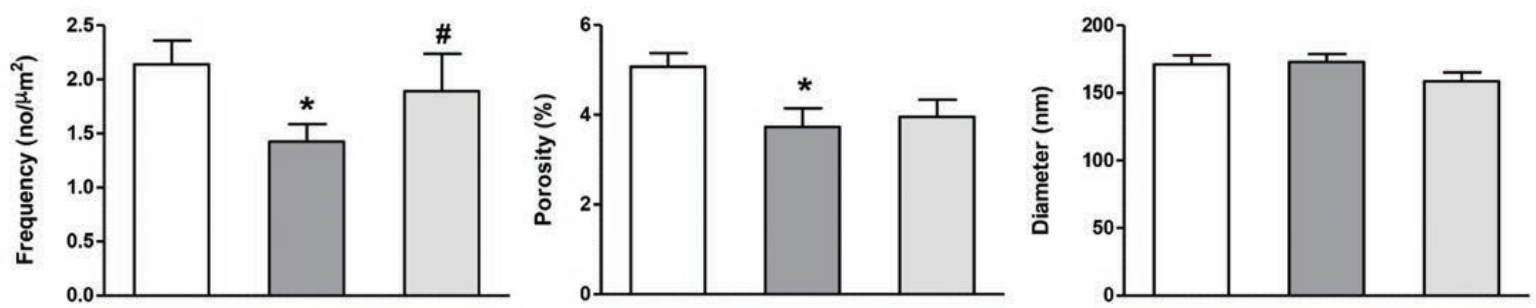

B
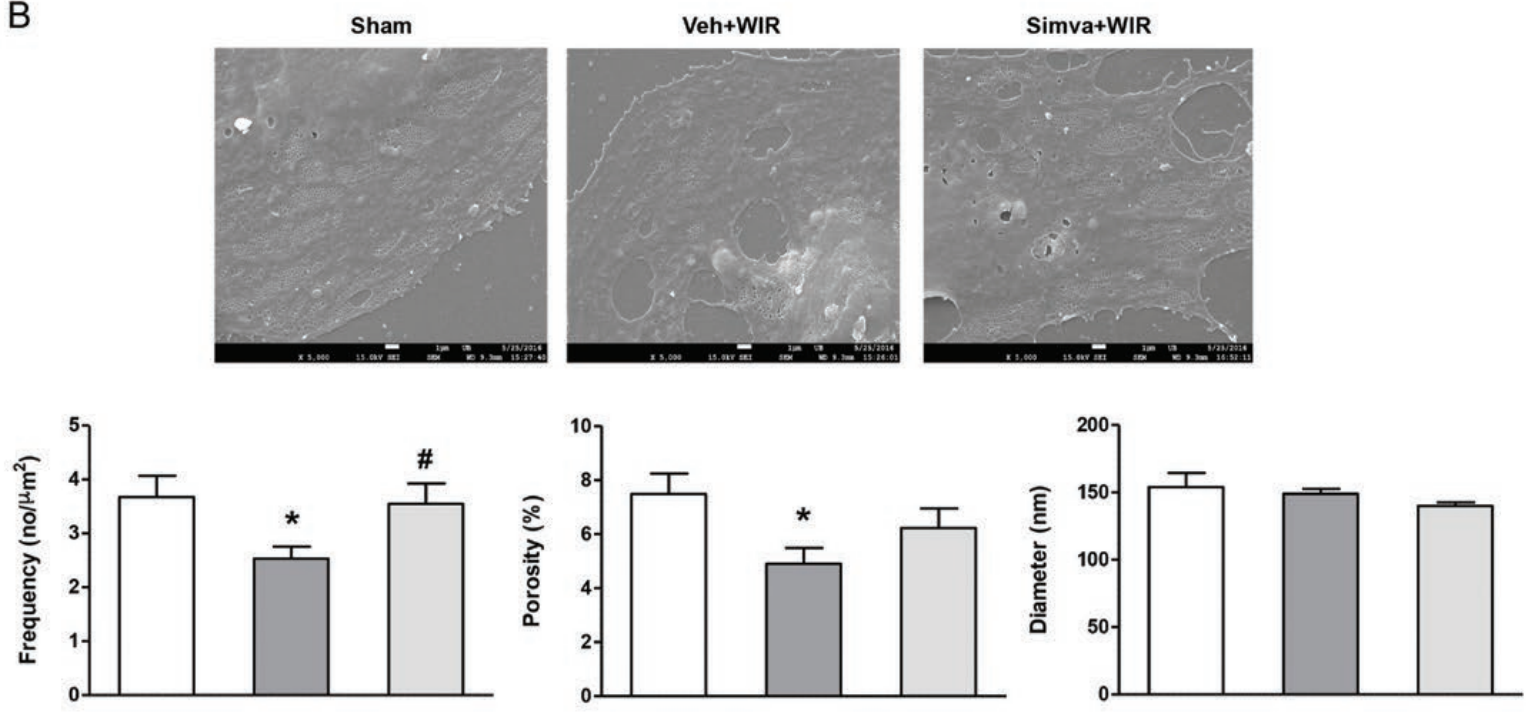

C

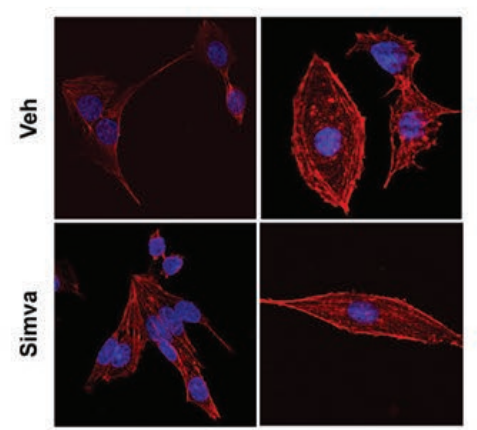

D
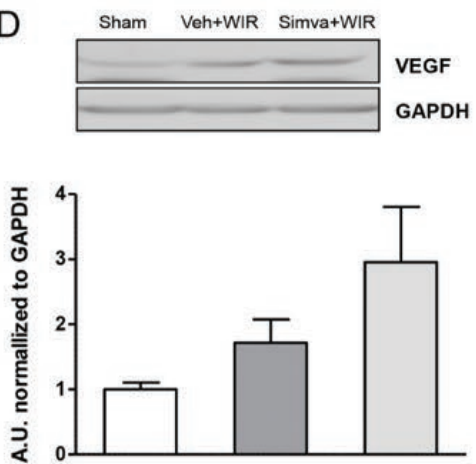

E
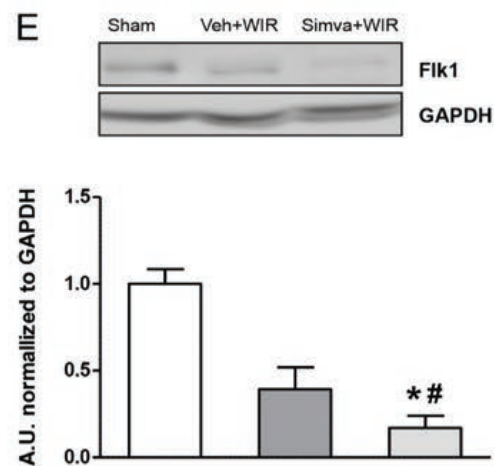

Figure 3. De-differentiation of the liver sinusoidal endothelium of aged livers suffering acute warm ischemia and reperfusion injury. (A) Liver tissue scanning electron micrographs from WIR-aged animals pretreated with simvastatin, or vehicle, or animals sham operated. Top: illustrative images representing the luminal surface of liver sinusoidal endothelial cells (original magnification $\times 15,000$ ). Bottom: fenestration frequency, porosity and diameter quantification. (B) Representative scanning electron micrographs of vehicle or simvastatin-treated LSEC undergoing anoxia/reoxygenation and quantification (original magnification $\times 5,000$ ). (C) Representative images of actin filament staining of cells described in B ( $\times 63$ ). (D) Representative images of hepatic VEGF immunoblots and densitometric analysis normalized to GAPDH from livers described in A. (E) Representative images of Flk-1 western blot and its corresponding quantification from livers described in A. $n=5$ animals per group (A, B, and C), $n=8$ animals per group (D-E); ${ }^{*} p<.05$ vs sham, ${ }^{*} p<.05$ vs vehicle). 
on liver function after treatment. Considering the vasoprotective effects of statins in young animals, we studied the effects of a 3-day pretreatment with simvastatin on liver endothelial function during WIR. Our results demonstrate that simvastatin diminishes the development of hepatic microcirculatory dysfunction in front of an acute injury in aged animals, which is accompanied by reduced parenchymal damage. We hypothesize that the beneficial effects of simvastatin on liver parenchyma are due to paracrine signals from the better-preserved sinusoids (41), although direct effects of the drug on hepatocytes cannot be discarded.

To unravel the underlying mechanisms causing WIR damage in aged livers we evaluated the NO pathway and ROS production as two main components regulating the hepatic microcirculation $(42,43)$. Our results reflect a burst in hepatic NO levels due to WIR that is accompanied by marked protein nitrotyrosination. Such increment in NO may be rarely due to a physiological NO production by eNOS, but to iNOS production. Indeed, it has been described that iNOS-derived NO results cytotoxic through the generation of reactive nitrogen species (RNS), promotion of proinflammatory cytokines, chemokines release, and leukocyte activation (44-47). We therefore analyzed the main enzymes contributing to hepatic NO generation and determined no change in iNOS expression but a significant reduction in phosphorylated eNOS in WIR-aged livers. Altogether suggesting that increased iNOS activity upon reperfusion is the main cause of NO boost, which will then result in ROS and RNS formation. While the role of the transcription factor Nrf2 was not conclusively shown, it would be interesting to follow up the role of this transcription factor in reducing the antioxidant capacity of those livers and how this may contribute to the increased oxidative stress observed.

Importantly, administration of simvastatin to aged rats was effective preventing the WIR-derived pathological increase in NO and protein nitrotyrosination, without modifying eNOS phosphorylation. The reduction in ROS formation was accompanied by a significant increase in hepatic Nrf2 protein expression and its target gene HO-1. These observations were complemented by the analysis of the specific phenotype of LSEC isolated from vehicle- or simvastatintreated aged animals that were then subjected to anoxia/reoxygenation in vitro mimicking in vivo WIR. The results support the data from liver tissue pointing to reduced WIR-derived ROS in LSEC from simvastatin-treated animals. Interestingly, these in vitro experiments revealed a trend of higher NO levels in endothelial cells from the simvastatin group, which may not be possible to observe when analyzing whole liver homogenates.

Finally, and as gold standard technique to evaluate the sinusoidal phenotype, we performed scanning electron microscopy in liver tissue and primary LSEC from the three experimental groups included in this study. Sham-operated animals had fenestrae frequency and porosity comparable to previous data (48), thus discarding microvascular injury due to animal manipulation. However, it is important to denote that just 1 hour of ischemia followed by 2 hours of reperfusion was enough to cause a significant decrease in fenestrae frequency and, therefore, reduced endothelial porosity without changes in fenestrae diameter. This detrimental effect of WIR was evident both in the sinusoidal endothelium of aged rats suffering acute liver injury in vivo, and in primary LSEC undergoing in vitro anoxia/reoxygenation. Interestingly, simvastatin administration before acute injury significantly prevented the deterioration in endothelial phenotype maintaining fenestrae frequency and porosity in both experimental conditions. Altogether, our results suggest that the vasoprotective effects of simvastatin would not only maintain LSEC functionality but also promote a better exchange of molecules between blood and hepatocytes, hence indirectly improving the phenotype of the liver parenchyma.

Although different research groups have focused on the study of fenestrae formation and modulation, to date no clear mechanistic description is universally accepted beyond the role of the cellular cytoskeleton in fenestrae dynamics (49). Consequently, we studied actin organization and part of its molecular mechanisms in LSEC isolated from aged animals showing a marked improvement in actin organization in response to simvastatin, which was accompanied by up-regulation in VEGF together with a negative feedback mechanism with its cellular receptor Flk-1. Another described mechanism implicated in fenestrae diameter regulation involves the activation of Rho protein family (including RhoA and Rho-kinase) and further phosphorylation of myosin light chain and inhibition of its phosphatase (36). Nevertheless, analysis of p-MLC and p-moesin in liver tissues from the vehicle and simvastatin WIR groups did not show any difference. The full characterization of fenestrae dynamics was out of the scope of the present study but future desirable experiments will further characterize the role of the cytoskeleton in LSEC fenestrae.

While we have used an experimental model of WIR that properly mimics common clinical situations such as hypovolemic shock consequence of a major hemorrhage, or hepatic ischemia and reperfusion occurring during the excision of small tumors, this model may not necessary extends to an accurate depiction of major hepatic resection post WIR. Future experiments analyzing the microcirculatory status at later reperfusion times may add important information to such clinical situations. Finally, it has to be noted that, although protective, the beneficial effects of statins in aged animals were less than those observed in young rats undergoing WIR (6). We cannot discard a possible optimization of simvastatin beneficial effects in the clinical scenario of acute liver injury in aged individuals by extending the period of treatment, likely after liver surgery. Indeed, unpublished data from our group demonstrate that 2-week simvastatin treatment improves chronic liver disease in aged animals (50). The limited results in old animals suggest that those individuals may require specific preclinical studies to develop safe and efficient treatments to prevent acute liver injury.

In conclusion, our study, although descriptive in nature, is the first revealing that aged livers are prone to develop sinusoidal microcirculatory dysfunction in response to an acute insult, which ultimately contributes to global liver damage. Consequently, considering the median age of patients undergoing hepatic surgery and the results herein described, we propose that future studies focused on the development of treatments for acute liver injury in the setting of ischemia reperfusion injury should include older animal models.

\section{Supplementary Material}

Supplementary data are available at The Journals of Gerontology, Series A: Biological Sciences and Medical Sciences online.

\section{Funding}

This work was supported by the Instituto de Salud Carlos III (FIS PI14/00029 and PI17/00012 to J.G.-S.), the European Union FEDER Funds, "una manera de hacer Europa," and the CERCA Program from the Generalitat de Catalunya. CIBEREHD is funded by the Instituto de Salud Carlos III. A.F.-I. has a Sara Borrell contract from the Instituto de Salud Carlos III (CD15/00050). R.M.-D. has a fellowship from the Instituto de Salud Carlos III (iPFIS IFI15/00037). 


\section{Acknowledgments}

This study was partly carried out at the Esther Koplowitz Center-IDIBAPS. Authors are indebted with Sergi Vila, Héctor García and Montse Monclús for technical assistance, and with the Electron Microscopy Unit (TEM/SEM) CCiTUB for assistance with the electron microscopy analyses.

\section{Authors Contributions}

D.H. designed the research, performed experiments, analyzed data, and wrote the article. A.W., A.F.I. and R.M.-D. performed experiments and analyzed data. C.P., D.G.L.C., J.B., and V.C.C. interpreted data and critically revised the article. J.G.-S. conceived the study, designed and directed the research, analyzed and interpreted data, wrote the article and obtained funding. All authors edited and approved the final article.

\section{Conflict of interest statement}

None reported.

\section{References}

1. Peralta C, Jiménez-Castro MB, Gracia-Sancho J. Hepatic ischemia and reperfusion injury: effects on the liver sinusoidal milieu. J Hepatol. 2013;59:1094-1106. doi:10.1016/j.jhep.2013.06.017

2. Jaeschke H. Mechanisms of liver injury. II. Mechanisms of neutrophilinduced liver cell injury during hepatic ischemia-reperfusion and other acute inflammatory conditions. Am J Physiol Gastrointest Liver Physiol. 2006;290:G1083-G1088. doi:10.1152/ajpgi.00568.2005

3. Abu-Amara M, Yang SY, Tapuria N, Fuller B, Davidson B, Seifalian A. Liver ischemia/reperfusion injury: processes in inflammatory networks-a review. Liver Transpl. 2010;16:1016-1032. doi:10.1002/lt.22117

4. Russo L, Gracia-Sancho J, García-Calderó H, et al. Addition of simvastatin to cold storage solution prevents endothelial dysfunction in explanted rat livers. Hepatology. 2012;55:921-930. doi:10.1002/hep.24755

5. Hide D, Ortega-Ribera M, Fernández-Iglesias A, et al. A novel form of the human manganese superoxide dismutase protects rat and human livers undergoing ischaemia and reperfusion injury. Clin Sci (Lond). 2014;127:527-537. doi:10.1042/CS20140125

6. Hide D, Ortega-Ribera M, Garcia-Pagan JC, Peralta C, Bosch J, GraciaSancho J. Effects of warm ischemia and reperfusion on the liver microcirculatory phenotype of rats: underlying mechanisms and pharmacological therapy. Sci Rep. 2016;6:22107. doi:10.1038/srep22107

7. Okaya T, Blanchard J, Schuster R, et al. Age-dependent responses to hepatic ischemia/reperfusion injury. Shock. 2005;24:421-427.

8. Selzner M, Selzner N, Jochum W, Graf R, Clavien PA. Increased ischemic injury in old mouse liver: an ATP-dependent mechanism. Liver Transpl. 2007;13:382-390. doi:10.1002/lt.21100

9. Clavien PA, Selzner M, Rüdiger HA, et al. A prospective randomized study in 100 consecutive patients undergoing major liver resection with versus without ischemic preconditioning. Ann Surg. 2003;238:843-850; discussion 851. doi:10.1097/01.sla.0000098620.27623.7d

10. Le Couteur DG, Rivory LP,Pond SM.The effects of aging and nutritional state on hypoxia-reoxygenation injury in the perfused rat liver. Transplantation. 1994;58:531-536. doi:10.1097/00007890-199409150-00001

11. Le Couteur DG, Tay SS, Solon-Biet S, et al. The influence of macronutrients on splanchnic and hepatic lymphocytes in aging mice. J Gerontol A Biol Sci Med Sci. 2015;70:1499-1507. doi:10.1093/gerona/glu196

12. Singh P, Goode T, Dean A, Awad SS, Darlington GJ. Elevated interferon gamma signaling contributes to impaired regeneration in the aged liver. $J$ Gerontol A Biol Sci Med Sci. 2011;66:944-956. doi:10.1093/gerona/glr094

13. Wang JH, Ahn IS, Fischer TD, et al. Autophagy suppresses age-dependent ischemia and reperfusion injury in livers of mice. Gastroenterology. 2011;141:2188-2199.e6. doi:10.1053/j.gastro.2011.08.005

14. Limani P, Linecker M, Oberkofler CE, et al. Remote ischemic preconditioning: a novel strategy in rescuing older livers from ischemia-reperfusion injury in a rodent model. Ann Surg. 2016;264:797-803. doi:10.1097/ SLA.0000000000001765

15. Wisse E, Braet F, Luo D, et al. Structure and function of sinusoidal lining cells in the liver. Toxicol Pathol. 1996;24:100-111. doi:10.1177/019262339602400114

16. Sørensen A, Mayntz D, Raubenheimer D, Simpson SJ. Protein-leverage in mice: the geometry of macronutrient balancing and consequences for fat deposition. Obesity (Silver Spring). 2008;16:566-571. doi:10.1038/ oby. 2007.58

17. Sørensen KK, McCourt P, Berg T, et al. The scavenger endothelial cell: a new player in homeostasis and immunity. Am J Physiol Regul Integr Comp Physiol. 2012;303:R1217-R1230. doi:10.1152/ajpregu.00686.2011

18. Fraser R, Cogger VC, Dobbs B, et al. The liver sieve and atherosclerosis. Pathology. 2012;44:181-186. doi:10.1097/PAT.0b013e328351bcc8

19. Hilmer SN, Cogger VC, Fraser R, McLean AJ, Sullivan D, Le Couteur DG. Age-related changes in the hepatic sinusoidal endothelium impede lipoprotein transfer in the rat. Hepatology. 2005;42:1349-1354. doi:10.1002/ hep. 20937

20. Le Couteur DG, Fraser R, Hilmer S, Rivory LP, McLean AJ. The hepatic sinusoid in aging and cirrhosis: effects on hepatic substrate disposition and drug clearance. Clin Pharmacokinet. 2005;44:187-200. doi:10.2165/00003088-200544020-00004

21. Maeso-Díaz R, Ortega-Ribera M, Fernández-Iglesias A, et al. Effects of aging on liver microcirculatory function and sinusoidal phenotype. Aging Cell. 2018:e12829. doi:10.1111/acel.12829

22. Jain MK, Ridker PM. Anti-inflammatory effects of statins: clinical evidence and basic mechanisms. Nat Rev Drug Discov. 2005;4:977-987. doi:10.1038/nrd1901

23. Abraldes JG, Rodríguez-Vilarrupla A, Graupera M, et al. Simvastatin treatment improves liver sinusoidal endothelial dysfunction in CCl4 cirrhotic rats. J Hepatol. 2007;46:1040-1046. doi:10.1016/j.jhep.2007.01.020

24. La Mura V, Pasarín M, Meireles CZ, et al. Effects of simvastatin administration on rodents with lipopolysaccharide-induced liver microvascular dysfunction. Hepatology. 2013;57:1172-1181. doi:10.1002/hep.26127

25. Tripathi DM, Vilaseca M, Lafoz E, et al. Simvastatin prevents progression of acute on chronic liver failure in rats with cirrhosis and portal hypertension. Gastroenterology. 2018;155:1564-1577. doi:10.1053/j. gastro.2018.07.022

26. Rodríguez S, Raurell I, Torres-Arauz M, García-Lezana T, Genescà J, Martell M. A nitric oxide-donating statin decreases portal pressure with a better toxicity profile than conventional statins in cirrhotic rats. Sci Rep. 2017;7:40461. doi:10.1038/srep40461

27. Casillas-Ramírez A, Zaouali A, Padrissa-Altés S, et al. Insulin-like growth factor and epidermal growth factor treatment: new approaches to protecting steatotic livers against ischemia-reperfusion injury. Endocrinology. 2009;150:3153-3161. doi:10.1210/en.2008-1458

28. Gracia-Sancho J, Laviña B, Rodríguez-Vilarrupla A, et al. Evidence against a role for NADPH oxidase modulating hepatic vascular tone in cirrhosis. Gastroenterology. 2007;133:959-966. doi:10.1053/j.gastro.2007.06.021

29. Le Couteur DG, Cogger VC, Markus AM, et al. Pseudocapillarization and associated energy limitation in the aged rat liver. Hepatology. 2001;33:537-543. doi:10.1053/jhep.2001.22754

30. Gracia-Sancho J, Russo L, García-Calderó H, García-Pagán JC, García-Cardeña G, Bosch J. Endothelial expression of transcription factor Kruppel-like factor 2 and its vasoprotective target genes in the normal and cirrhotic rat liver. Gut. 2011;60:517-524. doi:10.1136/ gut.2010.220913

31. Jiménez-Castro MB, Meroño N, Mendes-Braz M, et al. The effect of brain death in rat steatotic and non-steatotic liver transplantation with previous ischemic preconditioning. J Hepatol. 2015;62(1):83-91. doi:10.1016/j. jhep.2014.07.031

32. Wulf E, Deboben A, Bautz FA, Faulstich H, Wieland T. Fluorescent phallotoxin, a tool for the visualization of cellular actin. Proc Natl Acad Sci U S A. 1979;76:4498-4502.

33. BrandesRP,JaniszewskiM.Direct detection of reactive oxygen speciesexvivo. Kidney Int. 2005;67:1662-1664. doi:10.1111/j.1523-1755.2005.00258.x 
34. Gracia-Sancho J, Laviña B, Rodríguez-Vilarrupla A, et al. Increased oxidative stress in cirrhotic rat livers: a potential mechanism contributing to reduced nitric oxide bioavailability. Hepatology. 2008;47:1248-1256. doi:10.1002/hep.22166

35. Carpenter B, Lin Y, Stoll S, Raffai RL, McCuskey R, Wang R. VEGF is crucial for the hepatic vascular development required for lipoprotein uptake. Development. 2005;132:3293-3303. doi:10.1242/dev.01902

36. Yokomori H, Yoshimura K, Funakoshi S, et al. Rho modulates hepatic sinusoidal endothelial fenestrae via regulation of the actin cytoskeleton in rat endothelial cells. Lab Invest. 2004;84:857-864. doi:10.1038/ labinvest. 3700114

37. Zafra C, Abraldes JG, Turnes J, et al. Simvastatin enhances hepatic nitric oxide production and decreases the hepatic vascular tone in patients with cirrhosis. Gastroenterology. 2004;126:749-755. doi:10.1053/j.gastro.2003.12.007

38. Trebicka J, Hennenberg M, Laleman W, et al. Atorvastatin lowers portal pressure in cirrhotic rats by inhibition of RhoA/Rho-kinase and activation of endothelial nitric oxide synthase. Hepatology. 2007;46:242-253. doi:10.1002/hep. 21673

39. Abraldes JG, Villanueva C, Aracil C, et al.; BLEPS Study Group. Addition of simvastatin to standard therapy for the prevention of variceal rebleeding does not reduce rebleeding but increases survival in patients with cirrhosis. Gastroenterology. 2016;150:1160-1170.e3. doi:10.1053/j.gastro.2016.01.004

40. Helmy MM. Potential hepato-protective effect of $\alpha$-tocopherol or simvastatin in aged rats. Pharmacol Rep. 2012;64:698-705.

41. Ortega-Ribera M, Fernández-Iglesias A, Illa X, et al. Resemblance of the human liver sinusoid in a fluidic device with biomedical and pharmaceutical applications. Biotechnol Bioeng. 2018;115(10):2585-2594. doi:10.1002/bit.26776
42. Gracia-Sancho J, Marrone G, Fernández-Iglesias A. Hepatic microcirculation and mechanisms of portal hypertension. Nat Rev Gastroenterol Hepatol. 2018. doi:10.1038/s41575-018-0097-3

43. Iwakiri Y, Kim MY. Nitric oxide in liver diseases. Trends Pharmacol Sci. 2015;36:524-536. doi:10.1016/j.tips.2015.05.001

44. Billiar TR. The delicate balance of nitric oxide and superoxide in liver pathology. Gastroenterology. 1995;108:603-605. doi:10.1016/0016-5085(95)90093-4

45. Wang Y, Lawson JA, Jaeschke H. Differential effect of 2-aminoethyl-isothiourea, an inhibitor of the inducible nitric oxide synthase, on microvascular blood flow and organ injury in models of hepatic ischemia-reperfusion and endotoxemia. Shock. 1998;10:20-25.

46. Peralta C, Rull R, Rimola A, et al. Endogenous nitric oxide and exogenous nitric oxide supplementation in hepatic ischemia-reperfusion injury in the rat. Transplantation. 2001;71:529-536.

47. Shah V, Kamath PS. Nitric oxide in liver transplantation: pathobiology and clinical implications. Liver Transpl. 2003;9:1-11. doi:10.1053/ jlts.2003.36244

48. O'Reilly JN, Cogger VC, Le Couteur DG. Old age is associated with ultrastructural changes in isolated rat liver sinusoidal endothelial cells. J Electron Microsc (Tokyo). 2010;59:65-69. doi:10.1093/jmicro/ dfp039

49. Braet F, Wisse E. Structural and functional aspects of liver sinusoidal endothelial cell fenestrae: a review. Comp Hepatol. 2002;1:1. doi:10.1186/1476-5926-1-1

50. Maeso-Diaz R, Ortega-Ribera M, Lafoz E, et al. Aging influences hepatic microvascular biology and liver fibrosis in advanced chronic liver disease. Aging Dis. 2019. doi:10.14336/AD.2019.0127 